Mots. Les langages du politique

\title{
Évaluation de la présidentiabilité (à l'interface de la sémantique lexicale et de la linguistique de corpus)
}

Assessing présidentiabilité (at the interface point of lexical semantics to corpus linguistics)

Evaluación de la présidentiabilité (el interfaz entre la semántica lexical y la linguística de corpus)

Fabienne H. Baider

\section{OpenEdition}

\section{Journals}

Édition électronique

URL : https://journals.openedition.org/mots/22036

DOI : $10.4000 /$ mots.22036

ISSN : 1960-6001

Éditeur

ENS Éditions

\section{Édition imprimée}

Date de publication : 6 octobre 2015

Pagination : 103-128

ISBN : 978-2-84788-727-3

ISSN : 0243-6450

\section{Référence électronique}

Fabienne H. Baider, «Évaluation de la présidentiabilité (à l'interface de la sémantique lexicale et de la

linguistique de corpus)», Mots. Les langages du politique [En ligne], 108 | 2015, mis en ligne le 06 octobre 2017, consulté le 23 avril 2022. URL : http://journals.openedition.org/mots/22036 ; DOI : https://doi.org/10.4000/mots.22036 


\section{Évaluation de la présidentiabilité (à l'interface de la sémantique lexicale et de la linguistique de corpus)}

Nouvellement venus dans la langue française, le nom présidentiabilité et l'adjectif présidentiable ont été attestés selon le Trésor de la langue française informatisé (TLFi) en 1965 et en 1980 respectivement, les deux attestations provenant de discours journalistiques. Les définitions restent floues ${ }^{1}$ (présidentiable : qui est en mesure de se présenter comme candidat à la présidence selon le TLFi), cependant, le thème de présidentiabilité est devenu fondamental lors de l'évaluation des candidats présidentiels (Moureaud, 2008 ; Le Bart, 2009). Ce travail propose de cerner la construction dans la presse de cette thématique, essentielle pour le choix des électeurs, et par là même d'attester de l'importance de la linguistique de corpus dans l'étude d'une telle labellisation. Dans un premier temps, nous recherchons les associations lexicales les plus fréquentes avec le terme présidentiabilité pour déterminer les motifs de cette thématique (voir la proposition théorique de Cadiot et Vissetti). Dans un deuxième temps, nous travaillons avec des données focalisées sur Martine Aubry et François Hollande dans le quotidien Le Monde lors de la primaire socialiste de 2011 afin de tester la présence et l'importance de ces motifs dans les discours au sujet de ces personnes politiques ${ }^{2}$. Les notions de préférence et de prosodie sémantiques (Sinclair, 2004; Bednarek, 2008) sont mises en œuvre afin d'analyser l'axiologie positive ou négative des contextes dans lesquels sont ancrés les énoncés évaluant les deux personnes politiques. Avant de présenter ces données, nous faisons le point sur les travaux antérieurs consacrés à la notion de présidentiabilité ainsi que sur le cadre théorique et la méthodologie de linguistique de corpus adoptés.

1. Moureaud retrace les origines des termes (2008, p.161, notes 1 et 2.)

2. Même si Le Monde cite souvent les personnes politiques elles-mêmes, ces citations relèvent d'un choix du comité éditorial et nous les considérons donc comme faisant partie du discours sur les candidats. 


\section{Problématique, théorie, méthodologie}

\section{Problématique}

\section{Qu'est-ce que la présidentiabilité ? Un présidentiable?}

Nous considérons ici que la langue est « une activité auto-formatrice et un milieu constitué par sa nécessaire reprise et stabilisation à travers des mises en place thématiques» (Cadiot, Visetti, 2001, p. 2); de plus, nous posons que la mise en place de la thématique présidentiabilité et son évaluation sont le focus des discours journalistiques consacrés à des candidats à une élection présidentielle, la thématique étant définie comme étant «ce dont on parle» (ibid.).

Les définitions proposées du terme présidentiable insistent sur les aptitudes et la légitimité des candidats - voir par exemple Le Bart (2009, p. 40) : «[...] les candidats crédités par les grands médias d'une "capacité" et d'une "crédibilité" attestées [...] par l'investiture des grands partis, ou par les performances sondagières» (nous soulignons). Ces mêmes recherches - nous le constatons dans la citation ci-dessus - mettent aussi en avant le rôle conséquent des médias.

\section{La coconstruction discursive de la notion}

En effet, ces discours journalistiques et les médias en général sont une des ressources dans «l'émergence des présidentiables» (Moureaud, 2008, p.162) même si des réquisits, tels que l'expérience institutionnelle de haut niveau, la reconnaissance des pairs, et un soutien partisan doivent être remplis (Moureaud, 2008, p. 188-189) : les médias amplifient, légitiment et confirment ce statut et par là même, sous l'apparence de simplement rapporter des actes ou des propos, participent de l'objet et contribuent à la représentation (Bourdieu, 1988, p. 3).

Les quelques études qui ont travaillé sur la notion de présidentiabilité ont aussi mis au jour cette coconstruction de la notion (Moureaud, 2008, p. 188; Le Bart, 2009, p.40) par les différents acteurs de la vie politique : l'espace médiatique permet aux journalistes de (dis)qualifier les candidats de présidentiable(s) parce qu'ils rapportent et analysent les propos des autres commentateurs, des sondeurs et des politologues. La presse, parmi les autres médias, est donc un des lieux incontournables de la «labellisation» (Moureaud, 2008, p. 162), labellisation basée sur une évaluation.

\section{Évaluation, motif, thématique}

\section{Évaluation transversale et trame discursive}

Comme la présidentiabilité est a priori l'évaluation d'un présidentiable, notre étude est donc structurée par la multi-dimensionnalité de ce qui est 
nommé évaluation et notamment par la définition de R. Rezsohazy (2006) pour le concept de «valeur»3. Celui-ci aurait quatre dimensions : 1) un objet (ce qui est valorisé, apprécié) ; 2) un jugement sur cet objet; 3) des normes qui découlent de ce jugement; 4) les porteurs de valeurs ou acteurs sociaux. Dans cette étude :

- l'objet apprécié est ce qui constitue la présidentiabilité;

- le jugement favorable ou non quant à la présidentiabilité pourrait être estimé et cela fait partie de notre proposition, en observant la combinaison de la préférence et de la prosodie sémantiques des motifs 4 : ces choix linguistiques dépendent des auteurs de l'article, du comité éditorial et du choix politique du quotidien;

- les normes sont alors déduites de jugements; on peut aussi établir une relation entre les normes et les motifs, par exemple l'(in)expérience, l'(in)compétence (voir la section ci-dessous);

- les porteurs des valeurs attendues pour être qualifiés de "présidentiables » sont les acteurs politiques, en l'occurrence Martine Aubry et François Hollande dans cette étude. Le Monde est centre-gauche et son lectorat estimé être de gauche. Dans le cas de cette primaire, le quotidien avait déclaré son soutien à Martine Aubry.

Les unités textuelles étudiées sont au départ des articles de presse, mais l'analyse se fait à partir d'une lecture transversale de ces articles en isolant la «trame " présidentiabilité qui relie ces textes pour en faire le discours sur telle personne politique à un moment $X$ de l'histoire dans la course à la présidence en France.

\section{Motifs et thématique}

Nous considérons que cette évaluation discursive est construite à l'aide de motifs, c'est-à-dire de concepts qui font le thème, ainsi la capacité et la légitimité évoquées plus haut. Ces motifs sont toujours à définir puisqu'ils sont «des germes de signification chaotiques et/ou instables» (Cadiot, Visetti, 2001) et évoluent selon le cadre socio-culturel et historique. Ainsi, si la reprise de l'incapacité d'un candidat fait partie de la mise en place du thème de non-présidentiabilité, la définition même de capacité évolue selon le contexte socio-historique et la situation d'énonciation, les normes mises en jeu lors de l'évaluation, la position au sein du champ politique de l'énonciateur et les objectifs de cette évaluation. Un exemple précis est celui de l'adjectif gentil qui n'aurait pas sa valeur positive (voir TLFi : «Dont l'action est prévenante, délicate, agréable») dans nos données puisqu'il est redéfini contextuellement comme une qualité : «François Hollande est un homme gentil, et c'est une qualité » (Libération,

3. Travailler sur l'évaluation met en place différentes définitions et approches théoriques; ainsi en témoigne le numéro 184 de Langue française (2015) consacré à cette problématique.

4. Voir infra, section «Méthodologie», pour les définitions de ces termes 
13 juillet 2011) ; le terme est potentiellement aussi utilisé avec une pointe d'ironie puisque l'énonciateur, Laurent Fabius, ne soutenait pas François Hollande à l’époque, mais Martine Aubry.

\section{Méthodologie : transversalité, préférence sémantique, prosodie sémantique}

\section{Transversalité et saillance}

Nous proposons de détecter ces motifs et leur valeur dans les textes étudiés à partir de récurrences de syntagmes et de l'étude des cotextes de ces syntagmes (phrase ou paragraphe) à travers nos données. La transversalité qu'implique cette lecture complémente la proposition de Cadiot et Visetti, qui prône l'étude transphrastique comme le font les études qualitatives textuelles (Rastier, 2011; Rabatel, 1998). Nous estimons que la dimension de transversalité est importante dans le processus d'évaluation : en effet, la constance d'un discours favorable ou défavorable, que nous pouvons attester avec les dates des citations, intensifie l'évaluation par l'effet cumulatif, comme nous allons le voir dans cette étude5. Cette constance d'une valeur axiologique implique aussi une fréquence d'un certain champ lexical à valeur positive ou négative, dimension déterminante dans la saillance de tel ou tel trait (qui devient pertinent) pour une entité donnée (Giora, 2003).

Nous proposons d'évaluer les motifs (compétence, expérience, etc.) en établissant d'abord les affinités lexicales de ces motifs dans un corpus de référence $^{6}$ : par exemple, pour évaluer le motif expérience, nous travaillons à partir des collocations les plus fréquentes avec le terme expérience (ainsi habileté, connaissance, etc.). La présence (ou l'absence) de ces items linguistiques dans le discours sur les candidats ainsi que leur fonctionnement en discours détermineront le profilage du motif pour ce candidat. Le profilage est en effet le «système, déjà frayé et enregistré en lexique et en grammaire, de parcours de stabilisations différentielles pour des lexèmes en interdéfinition ». Les choix lexicaux et syntaxiques actualisent les motifs en discours et font partie du marquage évaluatif, polarisant négativement ou positivement les motifs (expérience vs inexpérience, légitimité vs illégitimité, etc.), et cela de manière plus ou moins intense.

\section{Préférence et prosodie sémantiques}

Les motifs pour chaque candidat seront interprétés à l'aide de deux concepts : la préférence sémantique et la prosodie sémantique (Sinclair, 2004; Bednarek, 2008)7. La préférence sémantique d'un terme fait référence

5. Ce serait ici plutôt l'intersectionnalité des motifs qu'un effet cumulatif (McCall, 2005).

6. Voir section «La présidentiabilité», infra.

7. Les collocats peuvent aussi avoir une valeur négative ou positive (Partington, 2004, et ce que 
aux collocats les plus fréquemment associés aux termes étudiés (collocational patterning pour Bednarek, 2008, p. 130) et aux champs sémantiques particuliers auxquels appartiennent les collocats (Sinclair, 2004)8 ${ }^{8}$ : par exemple la présidentiabilité d'une personne politique peut être associée plus fréquemment à l'expérience ou à la crédibilité.

De fait, les items linguistiques qui forment le motifs sont employés de manière récurrente dans un cotexte positif ou négatif; la récurrence connote alors de manière favorable ou défavorable la présidentiabilité de chaque candidat et consiste en la "prosodie sémantique». Celle-ci est déduite du fonctionnement pragmatique de la lexie dans l'énoncé (Sinclair, 2004, p. 34); c'est « un élément subtil qui a une signification pragmatique, et qui fait référence à l'attitude $»^{10}$. Dans le cas présent, nous proposons que la prosodie sémantique fasse référence à l'évaluation de l'énonciateur quant à la présidentiabilité des candidats. Ainsi la prosodie négative du motif compétence est construite dans nos données avec l'emploi d'adjectifs négatifs (bilan contestê), de déterminant négatif (aucune expérience, aucun charisme), du conditionnel quant à la réussite (gagnerait), etc. La prosodie sémantique dans ce travail est donc définie par l'existence de faisceaux d'items lexicaux et/ou de phénomènes (morpho)syntaxiques qui expriment une attitude (approbation, rejet, ironie, doute, etc.), construisant une évaluation positive, mitigée, négative, etc. de l'entité.

L'intersection des trois facteurs fréquence/constance dans le temps/axiologie positive ou négative résulte en un discours plus ou moins intensément favorable ou défavorable.

\section{Données, méthodologie, définition en contexte}

\section{Corpus, méthodologie}

Dans un premier temps, pour la définition de présidentiabilité, nous avons travaillé à partir de deux corpus. Le corpus de référence $A$ (ou corpus de contrôle) est celui de SketchEngine ${ }^{11}$ et a servi à l'étude quantitative nécessaire pour déterminer le profil (associations lexicales les plus fréquentes) de chaque motif. En effet, afin de cerner les éléments définitoires des concepts «présidentiabilité » et «présidentiable», nous n’avons trouvé que quelques

Bednarek marque «préférence POS et NEG», 2008). Nous ne travaillons que sur la valeur des cotextes et pas sur celle des collocats, puisque la valeur des collocats dépendra du cotexte et du contexte d'énonciation. Notre proposition est donc différente de celle adoptée par exemple par Grutschus et al. (2013), qui considèrent la prosodie sémantique au niveau des collocats.

8. [They collocate with] «items which share a semantic feature, for example that they are all about, say, sport or suffering» (Sinclair, 2004, p. 142).

9. Voir tableau 7 infra, section «Présidentiabilité dans les discours sur la primaire socialiste».

10. "Semantic prosody refers to attitudinal, often pragmatic meaning. " (ibid., p. 144-145)

11. http://www.sketchengine.co.uk/. 
cooccurrences de présidentiable ou présidentiabilité dans notre corpus B de moins de 2 millions de mots (2011-2012) et avons dû en effet travailler avec SketchEngine pour des résultats statistiques. Nous avons vérifié préalablement s'il était méthodologiquement recevable d'utiliser le corpus A pour des recherches ciblées en politique ${ }^{12}$. Ce corpus nous sert à cerner, à partir d'une étude qualitative, ce que sont les qualités recherchées d'un présidentiable pour cette campagne présidentielle.

Le corpus de référence $B$ rassemble des articles consacrés à tous les candidats de la présidentielle 2012 (mars 2011 - mai 2012, 1861340 mots, six quotidiens, tout bord politique). Nous avons extrait de ces données trois sous-corpus d'articles du quotidien Le Monde focalisés sur les deux présidentiables et comparables du point de vue de la taille.

\begin{tabular}{|c|c|c|}
\hline Le Monde & Nombre de mots & Période \\
\hline MARTINE AUBRY & 1. 246 & 1. MARS - 15 SEPTEMBRE \\
\hline FRANÇOIS HOLLANDE & 1. 765 & 1. MARS - 15 SEPTEMBRE \\
\hline FRANÇOIS HOLLANDE & 1. 470 & $1^{\mathrm{er}}$ avril $2012-6$ mai 2012 \\
\hline
\end{tabular}

Tenant compte de la temporalité de la campagne qui fait évoluer les positions, nous confrontons des données en mars-septembre et en avril-mai 2012 d'un même quotidien, qui idéologiquement pourrait soutenir l'un ou l'autre des candidats. Les deux premiers sont focalisés sur Martine Aubry et sur François Hollande avant la primaire socialiste et rassemblent pour chaque période au moins vingt-cinq articles ${ }^{13}$. Le deuxième est consacré à François Hollande avant le vote du 6 mai.

Nous testons dans les corpus focalisés sur les deux personnes politiques Aubry et Hollande les éléments définitoires trouvés à partir du corpus A. Nous repérons ainsi la fréquence des emplois lexicaux des motifs définitoires de la présidentiabilité (ainsi la compétence ou l'expérience), puis nous retournons aux concordances (le contexte) données par SketchEngine afin de repérer le jugement négatif ou positif de l'énonciateur lors de l'emploi de l'unité lexicale (prosodie sémantique).

\section{La présidentiabilité, être présidentiable}

\section{Évaluation de présidentiable et présidentiabilité?}

La base de données Sketchengine permet de déterminer un profil du mot ou wordsketch rassemblant toutes les parties du discours les plus fréquemment employés en collocation avec les noms travaillés.

12. Voir la section «La présidentiabilité, être présidentiable», infra.

13. Il est à noter que le corpus de Martine Aubry contient 30 articles et que celui de François Hollande en contient 25. Cela implique des articles plus longs en général sur ce dernier. 
Le mot présidentiabilité est trop rare pour recenser des fréquences. Il faut alors consulter les collocations les plus fréquentes avec présidentiable :

Tableau 1: Collocations les plus fréquentes avec présidentiable; contexte de + ou - 5 mots, fréquence, minimum de 5 occurrences (SketchEngine, 12 millions de mots)

\begin{tabular}{l|c|c|c|c}
\hline $\begin{array}{l}\text { Parties du } \\
\text { discours }\end{array}$ & Fréq. & T-Score & MI & logDice* $^{*}$ \\
\hline stature & 94 & 9694 & 13029 & 6712 \\
\hline carrure & 24 & 4897 & 11253 & 4899 \\
\hline galons & 11 & 3315 & 11329 & 4636 \\
\hline putatif & 5 & 2236 & 12936 & 4607 \\
\hline endossé & 6 & 2448 & 11254 & 4213 \\
\hline crédible & 39 & 6237 & 9650 & 3550 \\
\hline
\end{tabular}

*Nous prenons en compte la mesure statistique logDice, suivant en cela les recommandations émises dans les travaux de Cook et al. (2013).

Nos données 2011-2012, moindres que celles de SketchEngine, confirment néanmoins ces associations lexicales. Cela est important car ce constat valide le fait de travailler à partir de SketchEngine pour cerner les associations lexicales à la base de notre travail.

\section{Tableau 2.: Collocations les plus fréquentes avec présidentiable; contexte de + ou - 5 mots, fréquence, minimum de 3 occurrences (notre corpus journalistique, 1,8 million de mots)}

\begin{tabular}{l|c|c|c|c}
\hline $\begin{array}{l}\text { Parties du } \\
\text { discours }\end{array}$ & Fréq. & T-Score & MI & logDice* $^{*}$ \\
\hline habits & 3 & 1732 & 12000 & 10415 \\
\hline stature & 4 & 1999 & 10415 & 10206 \\
\hline crédible & 3 & 1730 & 9571 & 9574 \\
\hline possible & 3 & 1724 & 7830 & 8390 \\
\hline programme & 3 & 1718 & 6986 & 7681 \\
\hline projet & 4 & 1973 & 6230 & 7027 \\
\hline
\end{tabular}

Les associations lexicales au mot présidentiable dans les deux corpus pointent en effet de manière explicite : 1) la valeur attribuée au candidat (stature $^{14}$ qui est commun aux deux tableaux, carrure et habits), 2) la crédibilité, 3) le fait qu'être présidentiable est une notion qui se fait et défait (possible,

14. Selon le Trésor de la langue française informatisé : stature est la dimension, élévation, valeur que présente sur le plan moral une personne. 
putatif). La notion d'expérience est implicitement contenue dans galons (gagner, recevoir, prendre ses galons) et celle de compétence dans le mot stature mais aussi dans projet, programme.

De même, pendant la primaire, Laurent Fabius avait justifié son choix pour deux candidats (Dominique Strauss-Kahn et Martine Aubry) car ils avaient tous deux «l'expérience, la compétence et la légitimité » (Le Figaro, 11 avril 2011); ils étaient donc en mesure de se présenter comme candidats à la présidence.

Enfin, la citation ci-dessous hors corpus reprend ces trois dimensions de la présidentiabilité (expérience, compétences/aptitudes, caractère/stature) :

1. On se prend alors à faire l'inventaire de toutes les qualités d'un président idéal, contradictions incluses [...] : au cœur du tableau un caractère, une expérience, des compétences, une capacité à entraîner et à décider exceptionnels; enfin, une aptitude à la fois à être proche des Français et à tenir la dragée haute aux interlocuteurs étrangers de la France... ${ }^{15}$ (Nous soulignons)

Nous proposons donc de considérer les concepts de leadership, compétence, expérience et légitimité comme les motifs mis en place en discours pour évaluer la présidentiabilité des candidats en discours journalistique.

Afin d'établir le profil linguistique de ces concepts/motifs, nous repérons les champs lexicaux qui définissent ces termes en discours (fréquence en corpus). Faute de place, nous nous sommes limitée à la catégorie des noms et n'avons pris en compte ni les adjectifs ni les verbes ou adverbes, contrainte qui nous permettra tout de même de dresser une liste hypothétique de termes relatifs à l'évaluation des présidentiables. Les collocations listées sont limitées aux dix premières afin de nous en tenir aux items très courants.

\section{Leadership}

Les collocations les plus courantes pour définir le profil linguistique de leadership actualisent l'aptitude à diriger (management, gouvernance, stratégie, visionnaire, habileté) et la présentation de soi (charisme, professionnalisme, dynamisme, etc.). Ce concept est donc central à la présidentiabilité ; il recouvre à la fois les champs sémantiques de compétence et de stature ${ }^{16}$. Dans nos données, seuls les mots leader, charisme et gouvernance pourront être attestés; les autres items, s’ils sont présents, ne font pas référence aux candidats.

15. LesÉchos - http://www.lesechos.fr/01/06/2011/LesEchos/20945-076-ECH_presidentiabilite. htm (consulté le 11 mars 2015) : nous retrouvons dans cette citation les notions de proximité (être proche des Français) et d'aplomb (tenir la dragée haute) étudiées par Le Bart (2009) dans l'ethos des présidentiables Nicolas Sarkozy et Ségolène Royal durant la campagne de 2007.

16. Nous avons noté l'adjectif leadership féminin indiquant soit que le leadership est masculin par défaut, soit que les femmes gouvernent d’une manière différente. 
Tableau 3. Collocations les plus fréquentes avec leadership (SketchEngine)

\begin{tabular}{l|c}
\hline Noms & Log Dice \\
\hline management & 6,09 \\
\hline charisme & 6,01 \\
\hline dévouement & 5,51 \\
\hline gouvernance & 5,52 \\
\hline stratégie & 5,51 \\
\hline visionnaire & 5,15 \\
\hline professionnalisme & 5,07 \\
\hline créativité & 4,87 \\
\hline dynamisme & 4,75 \\
\hline
\end{tabular}

\section{Compétence}

Les résultats ci-dessous pour le motif compétence mettent en avant le jugement (gestion, évaluation, évaluer) et le savoir(-faire) (développement, savoirfaire, connaissance, expérience, bilan, etc.) :

Tableau 4. Collocations les plus fréquentes avec compétence (SketchEngine)

\begin{tabular}{l|c}
\hline Noms & Log Dice \\
\hline connaissances & 8,65 \\
\hline développement & 7,43 \\
\hline savoir-faire & 8,52 \\
\hline gestion & 7,20 \\
\hline bilan & 7,15 \\
\hline expérience & 7,15 \\
\hline formation & 6,64 \\
\hline évaluation & 7,05 \\
\hline habileté & 6,73 \\
\hline capacité & 6,67 \\
\hline
\end{tabular}

Dans nos données, seuls les mots gestion, bilan, expérience, capacité seront trouvés dans le cotexte des candidats.

\section{Légitimité}

Dans le corpus ${ }^{17}$, le mot légitimité, lorsqu'il fait référence à l'ethos et non au droit ou à une chose, est associé d'abord à crédibilité, représentativité, pertinence, respectabilité, efficacité.

17. Nous avons enlevé les termes qui se rapportaient soit à la légalité, soit aux différents types de pouvoir (monarchie, dynastie, etc.) 
Tableau 5. Collocations les plus fréquentes avec légitimité (SketchEngine)

\begin{tabular}{l|c}
\hline Noms & Log Dice \\
\hline crédibilité & 7,90 \\
\hline représentativité & 7,51 \\
\hline illégitimité & 6,30 \\
\hline pertinence & 5,99 \\
\hline respectabilité & 5,48 \\
\hline validité & 5,04 \\
\hline bien-fondé & 4,89 \\
\hline efficacité & 4,58 \\
\hline visibilité & 4,54 \\
\hline acceptabilité & 4,49 \\
\hline
\end{tabular}

\section{Expérience}

Les collocations les plus fréquentes mettent au jour un champ lexical différenciant l'expérience de la compétence et de la légitimé : celui du concret (savoir-faire, formation, pratique) et celui de la sagesse.

Tableau 6. Collocations les plus fréquentes avec expérience (SketchEngine)

\begin{tabular}{l|c}
\hline Noms & Log Dice \\
\hline savoir-faire & 8,37 \\
\hline connaissance & 7,94 \\
\hline compétence & 7,83 \\
\hline expertise & 7,71 \\
\hline savoir & 6,96 \\
\hline professionnalisme & 6,21 \\
\hline sagesse & 6,20 \\
\hline réflexion & 6,18 \\
\hline formation & 6,15 \\
\hline pratique & 6,02 \\
\hline
\end{tabular}

Les items linguistiques listés dans les tableaux de cette section seront considérés comme définissant les motifs constituant la présidentiabilité. Nous les avons repérés dans notre corpus d'articles de presse sur les deux personnes politiques afin de déterminer la présence ou non des motifs. Cela fait l'objet de la section suivante. 


\section{Présidentiabilité dans les discours sur la primaire socialiste}

Pour examiner, dans les données focalisées sur Martine Aubry et François Hollande, comment les motifs de compétence, expérience, leadership et légitimité structurent l'évaluation des protagonistes:

- nous tenons compte de la présence des items repérés dans la section précédente afin de déterminer la préférence sémantique des unités faisant référence aux présidentiables;

- nous examinons ensuite les passages contenant les unités repérées pour évaluer la prosodie sémantique des énoncés dans lesquels sont employées ces unités.

Nous observons ainsi une polarisation du discours pour ou contre les deux candidats résultant de l'intensité relative aux motifs, intensité résultant des effets cumulatifs de la préférence et de la prosodie sémantiques.

\section{Absence et présence des motifs ; préférence sémantique des noms Aubry et Hollande}

Nous avons limité notre recherche aux termes qui décrivaient uniquement les candidats ou leurs activités. De nombreux items lexicaux présents dans les tableauxn'ont pas été trouvés de manière explicite dans les corpus comme faisant référence aux candidats ${ }^{18}$ (management, dynamisme, professionnalisme, dévouement, etc.) ${ }^{19}$. Seuls 20 items linguistiques ont été attestés.

Le tableau ci-dessous présente le nombre d'occurrences en corpus pour chaque mot relatif à un motif, liste qui constitue le profil linguistique de la présidentiabilité dans les discours sur Martine Aubry et François Hollande. Nous employons les expressions entité Aubry et entité Hollande pour faire référence aux noms propres, aux expressions le candidat / la candidate, la première secrétaire du parti, la maire de Lille, le député de Corrèze, etc.

18. Ainsi le mot expert est présent dans les données car les candidats s'entourent d'expertise : «Depuis un an, Martine Aubry s'est préparée [...] constituant autour d'elle des réseaux d'experts, [...] » (24 mai 2011); "M. Hollande peut compter sur un réseau de deux cents experts» (1 er juillet 2011).

19. Toutes les notions inscrites dans les parenthèses sont présentes implicitement dans le texte; ainsi une accumulation de verbes peut-elle exprimer le dynamisme sans que les mots dynamique, dynamisme, n'apparaissent. Nous revenons dans notre conclusion sur la nécessité de compléter cette étude avec la prise en compte de l'expression implicite de ces notions. 
Tableau 7. Préférence sémantique pour les termes définissant la présidentiabilité

\begin{tabular}{|c|c|c|}
\hline & $\begin{array}{l}\text { Entité } \\
\text { Aubry }\end{array}$ & $\begin{array}{c}\text { Entité } \\
\text { Hollande }\end{array}$ \\
\hline \multicolumn{3}{|l|}{ Leadership } \\
\hline leader, leadership & 1 & 2 \\
\hline charisme, charismatique & 0 & 1 \\
\hline stratégie & 1 & 5 \\
\hline vision, visionnaire & 0 & 2 \\
\hline \multirow[t]{2}{*}{ gouvernance } & 1 & 0 \\
\hline & 3 & 10 \\
\hline \multicolumn{3}{|l|}{ Compétence } \\
\hline compétence & 0 & 1 \\
\hline bilan & 1 & 3 \\
\hline gestion/gérer & 1 & 0 \\
\hline habileté & 0 & 1 \\
\hline \multirow[t]{2}{*}{ capacité } & 3 & 1 \\
\hline & 5 & 6 \\
\hline \multicolumn{3}{|l|}{ Expérience } \\
\hline expérience & 3 & 7 \\
\hline \multirow[t]{2}{*}{ connaissance } & 0 & 1 \\
\hline & 3 & 8 \\
\hline \multicolumn{3}{|l|}{ Légitimité } \\
\hline légitime, légitimité & 6 & 0 \\
\hline \multirow[t]{2}{*}{ crédible, crédibilité } & 0 & 2 \\
\hline & 6 & 2 \\
\hline
\end{tabular}

Trois motifs sont polarisés entre les deux candidats de par une préférence sémantique contrastée. La préférence sémantique la plus marquée est leadership et expérience dans le cas de François Hollande et pour Martine Aubry, c'est légitimité. L'observation des contextes d'emplois des lexies repérées dans le tableau ci-dessus permettent de mettre au jour la mise en mots de ces unités lexicales.

\section{Concordances : prosodie sémantique des entités Aubry et Hollande}

\section{Le motif de leadership}

La préférence sémantique forte du motif de leadership pour François Hollande est accompagnée d'une prosodie sémantique peu favorable excepté dans ses qualités de stratège. En revanche, même si ce motif est moindre pour Martine Aubry, il est construit plus favorablement. 
Ainsi, si dans le cas de la candidate, son affirmation de leader est implicite en 2, le terme leader ${ }^{20}$ est explicitement nié dans le cas du candidat Hollande avec la forte présence d'adverbes de négation (n'a pas pris, n'est pas passé) et d'expression défavorable (un peu à la peine). En 4, en particulier, sont construits deux portraits opposés des deux candidats : l'une sûre d'elle et apparemment en tête et l'autre en mal, justement, de prendre la tête.

2. Le député des Landes met en avant la légitimité institutionnelle de la maire de Lille. "Quand un parti se choisit un ou une leader, il se choisit un candidat pour l'élection présidentielle. Cette conviction n'est pas variable selon les personnes", plaide-t-il. (19 mai 2011) ${ }^{21}$

3. "François Hollande n'a pas pris la place de DSK. Il n'est pas passé du statut de challenger à celui de leader», analyse un de ses visiteurs. (31 mai 2011)

4. La maire de Lille se prépare tranquillement, laissant son principal concurrent, François Hollande, un peu à la peine pour ajuster sa campagne et endosser le maillot de leader après la mise hors course de DSK. (18 juin 2011)

Quant à charisme, le terme n’est pas trouvé chez Martine Aubry et il est évalué négativement chez François Hollande (5):

5. «Le président dépeint volontiers M. Hollande comme un candidat «sympathique », mais sans le moindre charisme.» (29 juin 2011)

De même, dans les énoncés où paraissent le terme vision (qui ne semble pas présenter un plus chez aucun des candidats), la formule laisse explicitement de côté, en 10, présente la préoccupation européenne de François Hollande comme une tactique pour éviter la réalité (la primaire) ; la répétition de la fonction du candidat (député de Corrèze) en 6 et 7 pourrait aussi pointer un écart entre la fonction que François Hollande brigue et sa fonction actuelle.

6. Laissant explicitement de côté la primaire interne au Parti socialiste, le député de Corrèze a défendu sa vision de l'Europe et critiqué ses deux adversaires (10 mai)

7. En mettant notamment en avant «la vision réformiste et l'engagement européen » du député de Corrèze [...]. (1 ${ }^{\mathrm{er}}$ juillet 2011)

En 8, le parti aubryste s’inquiète du pari de leur leader, qui vise surtout les secteurs qu'elle sait être de son côté :

8. Sur l'efficacité de la méthode, le doute persiste. «Peut-être que c'est une vision

20. Le mot leader, dans les occurrences relevées, symbolise les qualités contenues dans leadership et n'est pas employé comme synonyme de représentant, chef, etc. À noter que le mot leadership n'est trouvé qu'en co-occurence avec Dominique Strausss-Khan. De plus, le motif compétence est principalement associé à cet homme politique et non pas à Martine Aubry ou à François Hollande; ex. : «Il apparaît comme le seul socialiste en capacité de préserver la gauche d’une élimination » (28 avril 2011). Les discours du Monde traduisent une hésitation envers les deux candidats «par défaut».

21. Les italiques dans les citations sont les nôtres pour mettre en avant les expressions importantes. 
un peu datée et qu'en segmentant, on prend le risque de ne pas donner une vue globale », s'inquiète un aubryste. (26 septembre 2011)

En ce qui concerne l'item stratégie, le profil est aussi mitigé pour les deux candidats. L'évaluation pour le candidat est parfois positive (en 9), puisqu'il consacre de l'énergie (accumulation de verbes travaille, propose, cultive), et qu'il est précis quant à son objectif (la différenciation). Cependant, elle est aussi fortement mise en doute en 10 et le discours énonciateur, en 11, semble ne pas prendre en charge les dires du candidat (jurant ne pas être).

9. Le député de Corrèze travaille, propose et cultive sa stratégie de différenciation. (2 avril 2011)

10. La stratégie de M. Hollande peut-elle fonctionner? «Le président normal, c'est sympathique, mais cela n'a jamais encore marché en France», déplore M. Bourlanges. (23 mai 2011)

11. Jurant ne pas être dans une stratégie de «débauchage», le député de Corrèze veut s'allier quelques poids lourds pour faire contrepoids à Mme Aubry dont il ne sous-estime pas la légitimité institutionnelle. (3 juin 2011)

En revanche, l'évaluation de la qualité de stratège pour Martine Aubry en 12, sans être négative, se base sur un ethos (dispose), sur un désir (se veut) et non pas sur une action ou un résultat:

12. Stratégie : Elle dispose de la légitimité institutionnelle, se veut la garante de l'unité des socialistes et du rassemblement de la gauche, l'incarnation d'un «pôle de stabilité». (20 mai)

Le leadership ne départage pas les deux candidats : tout d'abord, l'évaluation est mitigée pour les deux personnes politiques; ainsi, pour le candidat, ce motif serait plus construit autour de son habileté et de sa stratégie, alors que pour la candidate, ce serait sur ses capacités de gestionnaire. Il semblerait tout de même que la candidate Aubry soit la moins mauvaise jusqu'à la fin de l'été. Les inquiétudes quant à sa stratégie sont cependant prononcées fin septembre (12), au moment où les sondages ne sont plus en sa faveur.

\section{Évaluation de la compétence}

Le motif compétence ${ }^{22}$, plus fréquent dans les données du candidat François Hollande, ne présente pas non plus comme un avantage per se. Ainsi, une polarisation des compétences des deux candidats est révélée par la prosodie sémantique : l'évaluation positive du motif compétence repose plutôt, pour la candidate, sur ses capacités de gestionnaire (son bilan positif, ses capacités) et pour le candidat, plutôt sur son habileté comme c'était le cas pour leadership.

22. Nous prenons aussi en compte incompétence, inexpérience, etc. 
Le cotexte de bilan dans la citation 13 évalue favorablement les actions de la candidate (revendiquer la victoire, vote à l'unanimitê) dénotant la capacité d'action.

13. Dans son bilan, la première secrétaire peut revendiquer la victoire aux élections régionales et aux cantonales et le vote à l'unanimité du projet socialiste, pour lequel elle a réussi à associer tous les grands leaders du parti. (20 mai 2011)

Au contraire, en 14-16, les cotextes évaluent négativement la compétence de François Hollande (syntagmes adjectivaux bilan contesté, bilan peu glorieux) ; en 15-16, la négation d'un autre motif (manque d'expérience) intensifie le jugement négatif sur le candidat :

14. Les médias qu'il avait tant choyés dressaient un bilan peu glorieux de son règne. (2 avril 2011)

15. François Hollande ne dispose d'aucune expérience ministérielle et d'un bilan contesté à la tête du PS. (20 mai 2011)

16. Face à François Hollande dont elle raille le bilan à la tête du PS, elle compte sur son expérience ministérielle pour faire la différence. (7 octobre 2011)

On note cependant qu'en 16, à la fin de la primaire, c'est la candidate qui se moque du manque d'expérience de son adversaire alors qu'avant l'été, l'énonciation était prise en charge par d'autres politiques.

Le mot compétence, uniquement dans les données de François Hollande, est employé très négativement :

17. Les amis de DSK clament qu'il n'a ni l'expérience ni la compétence pour se présenter à la magistrature suprême. «François Hollande, dénoncent-ils, n'a jamais exercé de fonction ministérielle».

Au contraire, la seule occurrence de gestion dans les deux corpus évalue favorablement la compétence de la candidate; la référence au leadership (c'est la marque d'une femme d'État), intensifie le jugement favorable :

18. Depuis des semaines, elle montre que sa gestion du temps est la bonne. C'est la marque d'une femme d'État. (31 mai 2011)

Le cotexte du nom capacité évalue de manière mitigée Martine Aubry : positivement en 19, limitant la critique à ses adversaires en 20 et sans être débordant d'enthousiasme en 21, puisque c'est la capacité de travail qui est reconnue :

19. Confortée par le second tour des cantonales, même si la victoire est modeste, la maire de Lille compte faire la différence grâce à ses capacités de rassembleuse. (29 mars)

20. Réputée pour son mauvais caractère, ses adversaires s'interrogent sur ses capacités à rassembler. (24 mai 2011) 
21. "Elle a les défauts de ses qualités : c'est une femme de caractère qui a une énorme capacité de travail », assure Claude Bartolone. (24 mai 2011)

Dans le cas de François Hollande, c'est lui-même qui définit les qualités hypothétiques du candidat idéal :

22. François Hollande est convaincu que la primaire va se jouer sur la personnalité, sur la capacité à incarner la présidentialité et, en même temps, sur le lien avec les Français. (13 juillet 2011)

La stratégie de faire parler le candidat François Hollande pour affirmer ses propres qualités sera observée en filigrane dans les textes (ex. 22 et dans les sections ci-dessous). Cela peut indiquer la non prise en charge par l'énonciateur (Le Monde) de ces affirmations, et donc une distance par rapport au dire (positif). En revanche, dans le cas de Martine Aubry, ce sont d'autres politiques ou commentateurs qui mettent en avant ses qualités et non pas elle-même : ils apportent donc leur caution à la candidate par l'intermédiaire du quotidien.

La qualité qui fait la différence entre les deux candidats est l'habileté, absente des données de Martine Aubry, et évaluée favorablement pour François Hollande : d'un challenger, il est devenu un danger dans la course. Si cette habileté est circonscrite dans le temps (jusqu'à présent, face au suspens en 23), elle reste l'arme redoutable du candidat puisque son adversaire cherche à en faire de l'indécision (24). Nous verrons, dans la deuxième partie de ce travail, que le quotidien explique la victoire de François Hollande justement grâce à son habileté dans les relations humaines:

23. Jusqu'à présent, face au suspens autour de la candidature de Dominique Strauss-Kahn et de Martine Aubry, il a habilementoccupé le vide. (7 juin 2011)

24. Enfin, elle le tenait. Elle pensait bien l'avoir ferré, avoir déstabilisé sa tranquille assurance, démontré que son habileté cachait - mal - une incapacité à trancher. (17 septembre 2011)

En résumé, les compétences de la candidate sont celles de gestionnaire (gestion, bilan, capacités de travail) ; l'incompétence en la matière est aussi décrite pour le candidat. Les compétences du candidat sont celle de stratège et son habileté, notamment dans les relations humaines, compétences absentes dans le discours au sujet de Martine Aubry et même mises en doute (mauvais caractère en 20). Ont aussi été observées des différences dans les stratégies du quotidien pour présenter les qualités des candidats, stratégies qui semblent prendre une distance par rapport à François Hollande avant l'été mais qui semblent aussi remettre en cause Martine Aubry à partir de septembre.

\section{Évaluation du motif expérience}

Le discours sur la présidentiabilité de François Hollande est axé sur le motif expérience, étant donné la préférence sémantique élevée tout au long de la 
primaire. Cependant, les syntagmes nominaux et leurs cotextes évaluent très négativement ce motif en 25-30: l'accumulation de l'intensité d'un échec passé (il fut balayê), la répétition de son manque «total» d'expérience dans la plupart des occurrences, les prises de mauvaises décisions (il a fait une erreur) tracent un portrait catastrophique du candidat en la matière :

25. Son expérience de 2006, où il fut balayé avec Laurent Fabius par la tornade Ségolène Royal [...] (29 mars 2011)

26. Les amis de DSK clament qu'il n'a ni l'expérience ni la compétence pour se présenter à la magistrature suprême. (29 mars 2011)

27. François Hollande ne dispose d'aucune expérience ministérielle. (20 mai 2011)

28. M. Hollande, lui, est raillé [...] et pour «son manque d'expérience», alors qu'il n'a jamais été ministre. (29 juin 2011)

29. L'UMP raille [...] l'« inexpérience» de François Hollande. (29 juin 2011)

30. Hollande a fait une erreur avec la présidence normale et n'a aucune expérience. (29 juin 2011)

Qui plus est, son manque d'expérience est comparé à l'expérience diverse et importante de son adversaire Martine Aubry (31-33).

31. François Hollande apparaît moins armé que sa rivale sur le plan de son expérience ministérielle. (3 juin 2011)

32. La candidate compte faire valoir son bilan à la mairie de Lille, mais aussi son expérience chez Pechiney. (13 juillet 2011)

33. Elle compte sur son expérience ministérielle pour faire la différence. (7 octobre 2011)

De plus, l'évaluation de Martine Aubry est uniquement défavorable en ce qui concerne une expérience de campagne électorale (34), élément qui pourrait être en résonance avec son évaluation moyenne de stratège. On remarque que cette évaluation négative est aussi restreinte aux dires de ses adversaires et donc pourrait délégitimer cette attaque, qui semble «contenue» :

34. Ses adversaires soulignent à l'envi son manque d'expérience électoral. (29 juin 2011)

L'autre facette du motif expérience, la connaissance (de la France), est mentionnée uniquement dans le cas de François Hollande, qui parle de lui-même en 35 pour se défendre des accusations de manque d'expérience dans les fonctions ministérielles:

35. L'intéressé oppose sa maîtrise des dossiers et sa connaissance de la France, parcourue sans relâche depuis vingt ans. (29 mars 2011)

Le discours construisant le motif expérience révèle donc une évaluation déficitaire dans le cas de François Hollande. Alliée à la forte préférence sémantique 
de ce motif, l'évaluation, de par sa constance dans le temps et sa polarité clairement affirmée, devient fortement négative. En revanche, dans les données de Martine Aubry, ce motif est affirmé.

\section{Évaluation du motif légitimité}

Le motif légitimité a une préférence sémantique forte pour le discours relatif à la candidate, faible chez le candidat. La prosodie sémantique est aussi largement positive en ce qui concerne Martine Aubry (36-40), ainsi les cas cidessous de légitimité (elle dispose de la légitimité, on relève ou juge qu'elle a la légitimité; elle joue pleinement de sa légitimité) qui est reconnue et mise en avant par d'autres que la candidate :

36. [...] ils jugent qu'elle a la légitimité pour rassembler tous les socialistes et audelà toute la gauche (30 mars 2011)

37. Elle avait compté sur les cantonales, pour asseoir sa légitimité. (28 avril 2011)

38. Le député des Landes met en avant la légitimité institutionnelle de la maire de Lille. (19 mai 2011)

39. Elle dispose de la légitimité institutionnelle. (20 mai 2011)

40. Bernard Soulage relève la "légitimité institutionnelle de la première secrétaire». (24 mai 2011)

41. Martine Aubry a joué pleinement auprès des socialistes de sa légitimité institutionnelle. (29 juin 2011)

L'association de la préférence sémantique constante dans le temps, et de la prosodie sémantique positive résulte en une évaluation fortement positive de ce motif pour Martine Aubry. Le mot est absent des données du candidat. Nous pouvons aussi observer la diversité des désignations pour la candidate (maire de Lille, première secrétaire) par rapport aux répétitions du syntagme député de Corrèze (François Hollande était aussi ex-secrétaire du parti, par exemple).

En qui concerne l'item crédibilité, absent des données de Martine Aubry, l'énoncé 42 mentionne l'habileté du candidat à avoir perçu la demande des votants mais en 43 il avoue devoir retrouver la crédibilité, ce qui peut laisser supposer que la garantie du sérieux évoquée dans la citation était aussi absente de son ethos'23:

42. "La percée de Hollande existe mais elle est relative. Il occupe un vide. Il a perçu une demande de crédibilité», souligne un proche de Dominique Strauss-Kahn. (17 avril 2011)

43. Je devais apporter la garantie du sérieux, retrouver de la crédibilité. Mon image était celle d'un homme de la synthèse. (2 avril 2011)

23. Ce qui est le cas puisque la fin de la citation l'explicite : «J'étais enfermé dans ce personnage humoristique, sympathique et rond, devenu caricatural. Je devais être plus anguleux, plus vertébré, brefavoir une présidentiabilité.» 
En conclusion de cette partie :

1. Les données concernant la candidate Martine Aubry se caractérisent :

- par le motif leadership peu développé et un discours mitigé à ce sujet ;

- par le motif compétence affirmant ses capacités de gestionnaire, mais un déficit dans les facultés de stratège;

- par le motif légitimité important (préférence sémantique élevée) et une prosodie sémantique positive affirmant cette dimension dans sa candidature;

- par le motif expérience peu développé mais connaissant une prosodie sémantique positive.

Ces deux dernières conclusions pointent une évaluation plutôt favorable de la présidentiable.

2. Les données concernant le candidat François Hollande présentent :

- une préférence sémantique élevée pour le motif leadership et une prosodie sémantique mitigée : il serait possiblement un stratège, mais sans charisme;

- un motif compétence fortement déficitaire en gestion mais positif en habileté politique;

- une préférence sémantique élevée et une prosodie sémantique très négative associée au motif expérience, révélant ainsi la principale faiblesse de la candidature;

- une quasi-absence du motif légitimité, mis en doute de nouveau à cause de l'inexpérience.

Ces observations mènent à la conclusion d'une évaluation de la présidentiabilité plus favorable dans le cas de Martine Aubry que dans celui de son adversaire François Hollande. De plus, l'évaluation qui se dégage de nos observations s'aligne sur les accusations formulées par la candidate à l'encontre son adversaire : "niveau zéro en politique» (motifs de légitimité et expérience), «gauche molle» (motif de leadership) (L'Express, 12 octobre 2011)24.

\section{Après la primaire : de la gauche molle à la force tranquille?}

Nous avons procédé à la même étude afin d’identifier un possible «ajustement » des motifs après la victoire de François Hollande sur son adversaire Martine Aubry. En effet, si «la stratégie de campagne de François Hollande vise à corriger un déficit de stature présidentielle» (La Croix, 17 avril 2012), celle du Monde vise-t-elle les mêmes objectifs dans le corpus avril-mai?

24. http://www.lexpress.fr/actualite/politique/hollande-aubry-de-si-mauvais-camarades _1039303.html, consulté le 11 mars 2015. 


\section{Préférence sémantique du nom Hollande}

\section{Une volte-face discursive}

Comme le notent les quotidiens ainsi le journal Ouest France (12 avril 2012), le motif de mollesse est devenu celui de force tranquille dans le discours de Martine Aubry :

44. La maire de Lille et patronne du PS lance dorénavant des adresses inconditionnelles «à François auréolé de toutes les qualités»: "Ton engagement, ta détermination, ta force tranquille, ta dignité me rendent fière d'être socialiste! Tu es le porte-drapeau du peuple, tu portes ses espoirs et lui, je le sais, te portera à l’Élysée!»

Ce qui fera dire au journal La Croix (17 avril 2012) : «À l'heure de la revanche du «petit mécano », rares sont les socialistes à contester aujourd'hui son autorité», et amènera d'autres journaux à reprendre l'ethos de fermeté affiché par le candidat :

45. «Donc j'aurai, si les Français m'en donnent la capacité par le suffrage, à ouvrir cette discussion ferme et amicale avec Madame Merkel», a-t-il prévenu. (Libération, 26 avril 2012)

46. François Hollande s'est montré nettement plus ferme que le PS sur cette question. (Ouest-France, 4 mai 2012)

Dans le discours du Monde, on s'interroge sur cette volte-face discursive :

47. Le candidat par défaut serait-il devenu le candidat sans défauts? (3 mai 2012)

Pourtant, le tableau 8 reportant les occurrences des motifs dans le discours du Monde en avril-mai 2012 atteste aussi un repositionnement du discours dans ce quotidien, ainsi la quasi-disparition des motifs évalués défavorablement. Cependant, le même tableau ne présente pas non plus un gain substantiel sur les motifs qui font un présidentiable, même si nous prenons en compte que ce corpus compte un tiers de mots en moins par rapport aux données de la primaire. 
Tableau 8. Comparaison de la préférence sémantique du nom Hollande avant et après la primaire

\begin{tabular}{l|c|c}
\hline & $\begin{array}{c}\text { mars-juillet } \\
\text { nombre d'occurrences }\end{array}$ & $\begin{array}{c}\text { avril-mai } \\
\text { nombre d’occurrences }\end{array}$ \\
\hline Compétence & 1 & 0 \\
\hline compétence & 1 & 2 \\
\hline capacités & 3 & 0 \\
\hline bilan & 1 & 0 \\
\hline habileté & 0 & 2 \\
\hline gestion, gérer & 6 & 4 \\
\hline Expérience & 1 & 0 \\
\hline expérience & 8 & 0 \\
\hline connaissance & & 0 \\
\hline Légitimité & 0 & 1 \\
\hline légitime, légitimité & 2 & 0 \\
\hline crédible, crédibilité & $\mathbf{2}$ & 1 \\
\hline
\end{tabular}

Si la préférence sémantique atténuée a pour conséquence une baisse d’intensité dans le discours négatif (moins fréquent, moins constant), la prosodie sémantique des motifs n'accuse pas un changement de polarité.

\section{Un discours « légitimant»?}

\section{Motif leadership}

Le motif leadership, déficitaire lors de la primaire, se réduit à une attaque de Nicolas Sarkozy contre François Hollande :

48. Accuser son adversaire d'adopter une stratégie de «l'esquive» avait déjà été un angle d'attaque de l'UMP avant le premier tour. (10 avril 2012)

\section{Motif compétence}

Le motif compétence connaît encore une prosodie négative : en 49, le discours énonciateur garde la distance relevée lors de la primaire quant aux qualités du candidat, ainsi les expressions son candidat (on aurait pu choisir $d u$ candidat), pour la première fois (ce qui semble tardif à quelques semaines du vote), lâche (verbe employé pour des propos « inconvenants » selon le TLFi, ou du moins pour des aveux qui sont surprenants). L'expression s'attache à faire indiquerait que des éléments présentés comme des qualités dans la phrase précédente sont en fait des capacités en devenir : 
49. Partisan convaincu du oui, Pierre Moscovici préfère vanter les qualités de son candidat, «qui réconcilie, qui redonne à ce pays le goût de l'unité ». Ce que M. Hollande s'attache à faire, qui, pour la première fois, lâche: «Je suis socialiste, mais je me sens écologiste». (12 avril 2012)

À la veille du scrutin, les doutes restent :

50. Ses défauts deviennent des qualités : l'homme qui consulte à tout-va avant de se décider, n'est-ce pas la preuve d'une forme de sagesse plutôt qu'une faiblesse? À quoi servent toutes ces lois votées à la hussarde qui ne seront jamais appliquées? (4 mai 2012)

Les défauts de gestionnaire du candidat sont toujours la cible du discours, que ce soit pour son bilan ou sa gestion catastrophique, ainsi la citation 51 décrivant l'historique du parcours politique du candidat, ce qui n'aide pas la campagne de François Hollande :

51. Décembre 2008 [...] Érigé en cible, pendant les hostilités, par toutes les écuries en lice, tant pour sa méthode de la «synthèse molle» que pour le bilan de ses onze années rue de Solférino, François Hollande est désormais quotidiennement éreinté par Martine Aubry, qui lui succède [...] Laquelle envisage même un «audit » sur sa gestion et se répand, jusque devant le bureau national, sur l'état désastreux des toilettes... (24 avril 2012)

La seule occurrence pointant la force tranquille (ainsi sans dévier de sa ligne en 52) limite ses qualités à celle de gestionnaire de campagne, et reprend en écho l'habileté politique énoncée lors de la primaire :

52. "Ça n'a pas été qu'une partie de plaisir. Il y a eu des moments éprouvants", lâche François Hollande. Jusqu'au bout, il aura mené campagne en gestionnaire de son avance sondagière, sans dévier de sa ligne. (24 avril 2012)

\section{Motif légitimité}

Le motif légitimité se réduit encore à l'occurrence de crédibilité comme lors du premier corpus, et la prosodie sémantique est négative (n'apprécie guère, reproche, un manque de crédibilité internationale) :

53. Alain est de gauche "depuis toujours», mais cette année, il a voté pour François Bayrou car il n'apprécie guère $\mathrm{M}$. Hollande à qui il reproche un manque de «crédibilité internationale». (4 mai 2012)

En conclusion de cette partie :

- ni la préférence sémantique, ni la prosodie sémantique n’atteste une évaluation favorable dans le discours du Monde;

- même s’il a été identifié un évitement des motifs déficitaires de présidentiabilité, ce choix prend de la distance par rapport à d'autres discours journalistiques qui s'attachent de manière plus évidente à construire une force tranquille ; 
- l'évaluation du présidentiable vise plutôt à déconstruire les motifs de présidentiabilité de l'adversaire Nicolas Sarkozy, mettant par exemple en doute sa compétence. Ainsi, la préférence sémantique de bilan (appartenant au motif compétence) est plus élevée pour Nicolas Sarkozy que pour François Hollande et la prosodie sémantique est très négative :

54. Nicolas Sarkozy pratique en catastrophe la surenchère, sur un sujet où ses promesses sont en réalité un véritable désaveu de son bilan. (10 avril 2012)

55. [...] son bilan «nul», «dramatiquement négatif», bref, «un fiasco» (11 avril 2012).

56. À l'autre bout de la France, les flèches que décoche le candidat PS sur le bilan du président sortant font mouche également. (4 mai 2012)

De fait, la citation ci-dessous résume les conclusions quant à l'attitude du quotidien :

57. Après l'homme providentiel (DSK), il va falloir se contenter de l'homme normal. La tortue François Hollande, partie avant les autres dans l'indifférence générale et les railleries, va bientôt arriver au terme du chemin. (4 mai 2012)

Donc, nous proposons que le discours du Monde semble avoir adopté un compromis discursif à deux reprises :

- lors de la primaire, le champion qui était Dominique Strauss-Khan ayant été mis hors course, le discours du Monde fait «contre mauvaise fortune bon cœur» et semble soutenir Martine Aubry;

- lors du scrutin, le discours se focalise sur les défauts de l'adversaire plutôt que sur les qualités de François Hollande, ne redéfinissant pas le motif de mollesse, prégnant dans le discours sur le candidat lors de la primaire, en une force tranquille afin de (re)légitimer la présidentiabilité du candidat, ce que d'autres quotidiens semblent avoir fait.

\section{Remarques finales}

\section{Implications sociopolitiques de cette étude}

Les observateurs de la campagne présidentielle savent que le quotidien n'a endossé la candidature de François Hollande que très tardivement, et apparemment ne voulait pas désavouer son choix lors de la primaire. Il semble d'ailleurs que la position politique du Monde ne soit pas en porte à faux avec celle d'autres médias : dans la base de SketchEngine de 12 millions de mots, les noms, dans le cotexte de l'adjectif présidentiable, sont ceux des personnes politiques de la campagne présidentielle 2012. Sont cités d'abord le nom Aubry (5 occurrences) et ensuite Hollande ( 4 occurrences) : les occurrences de Aubry 
sont favorables (SketchEngine : «Très souriante, galvanisée par un sondage qui la conforte dans un statut de présidentiable, Martine Aubry a fustigé «l'inefficacité», [...] de Nicolas Sarkozy ») alors que celles de Hollande sont mitigées (SketchEngine : «Tout simplement François Hollande, propret présidentiable du Parti socialiste-sans-rire... »).

Cette étude confirme donc le poids partisan et celui des sondages dans l'évaluation définitive des candidats car, comme l'ont précisé les spécialistes du discours politique, la «crédibilité » serait attestée, entre autres, "parl'investiture des grands partis, ou par les performances sondagières » (Le Bart, 2009, p.40). De fait, François Hollande a gagné la primaire avec la volte-face de certains ténors socialistes (ainsi celui de Arnaud Montebourg) 25 mais aussi avec l'appui des sondages, dont Martine Aubry ne bénéficiait plus, juste avant le scrutin de la primaire. Cependant la personnalité des candidats reste un atout (ou un problème) non négligeable. Selon le quotidien, le candidat avait su « très habilement définir devant Arnaud Montebourg l'alliance qu'il comptait établir avec lui dans l'entre-deux tours» (Le Monde, 20 octobre 2011). La personnalité, l'habileté politique, François Hollande lui-même les avait prédites comme facteurs déterminants pour le vainqueur : «Cela va se jouer sur les personnalités, les alliances, la capacité à rassembler, et à incarner la fonction suprême » (Le Monde, 3 juin 2011). Et tout au long de cette étude, François Hollande avait été évalué plus favorablement que Martine Aubry sur ce point. Enfin, il est à remarquer le double sens possible de la formule choisie par François Hollande pour sa campagne : «être un président normal». La plupart des commentateurs ont analysé cette devise comme adressée au président sortant Nicolas Sarkozy, connu pour sa personnalité peu protocolaire. Cependant la formule, employée dès le début de la primaire, peut tout aussi bien s'adresser à Martine Aubry, qui de toute évidence, en tant que femme, ne peut incarner la normalité dans un système où aucune femme politique n'a jamais atteint cette fonction «suprême ». Ainsi, d'une pierre, François Hollande atteignait deux adversaires.

\section{Implications linguistiques}

Sur le plan linguistique, cette étude nous a permis tout d'abord de proposer la prise en compte du rôle de la transversalité dans le discours évaluatif, transversalité qui fait référence à la construction «au fil des textes» d'une évaluation favorable ou défavorable, le nom propre ou les références aux présidentiables se chargeant des effets cumulatifs de ces cooccurrences (Hoey, 2003). Même si les études fines de la coconstruction de l'évaluation transphrastique

25. "Arnaud Montebourg tente de rassurer ceux qu'il a déçus » : Le Monde, 20 octobre 2011, http:// www.lemonde.fr/primaire-parti-socialiste/article/2011/10/20/arnaud-montebourg-tente-derassurer-ceux-qu-il-a-decus_1590790_1471072.html\#K6Tb4Aq9AyYrZkbc.99 (consulté le 11 mars 2015). 
seront toujours indispensables pour compléter notre proposition, cet aspect évaluatif ne semble pas pouvoir être négligé lors de l'étude de discours électoraux, ancrés dans des notions « slogans » qui se construisent transtextuellement dans le but de «labelliser» les candidats favorablement.

Ensuite, notre lecture s'est basée à la fois sur la notion de préférence sémantique, identifiable semi-automatiquement, et sur la prosodie sémantique repérée manuellement dans des cotextes, eux-mêmes identifiables semi-automatiquement. Nous avons proposé d'interpréter la notion de prosodie sémantique, notion qui reste floue selon plusieurs auteurs, comme l'axiologie positive ou négative du cotexte dans lequel sont ancrées les entités Aubry et Hollande tout au long de la primaire et cela de manière constante. Une prosodie sémantique favorable/défavorable de manière consistante dans le temps, alliée à une préférence élevée, pointe alors une intensité positive/négative d'un motif pour les candidats dans le positionnement des énonciateurs quant à ces candidats.

Enfin, nous avons aussi pu faire émerger des catégories évaluatives de la thématique présidentiabilité à partir du regroupement d'items lexicaux les plus fréquents, conformément au principe de saillance définie dans Giora (2003).

\section{Pistes à poursuivre}

Il va sans dire que nous avons formulé des hypothèses, mais que celles-ci doivent aussi être vérifiées avec, entre autres :

- l'étude paradigmatique de chaque motif, ainsi la présence non pas seulement de leadership mais aussi de femme ou homme d'État; non pas seulement de capacités mais aussi de être capable, avoir l'aptitude, etc. ;

- la prise en compte de l'expression indexicale des motifs, notamment par les verbes ou les adjectifs, que nous avons aussi repérés mais qui ne pouvaient faire l'objet de ce travail, ainsi que celle des structures syntaxiques, en particulier l'observation de la place des noms des candidats dans les clauses enchâssées, et leur fonction grammaticale;

- l'étude de la construction de la présidentiabilité au fil du texte et non plus au fil des textes.

\section{Références}

BedNAREK Monika, 2008, "Semantic preference and semantic prosody re-examined ", Corpus Linguistics and Linguistic Theory, $\mathrm{n}^{\circ} 4$ (2), p. 119-139.

Bourdieu Pierre, 1988, "Penser la politique», Actes de la recherche en sciences sociales, $n^{0} 71-72$, p. 2-4.

CADIOT Pierre, VISETtI Yves-Marie, 2001, «Motifs, profils, thèmes. Une approche globale de la polysémie», Cahiers de Lexicologie, nº 79, p. 5-46. 
Cook Paul, HAN LAU Jey, Rundell Michaël, McCARThy Diana, Baldwin Timothy, 2013, «A lexicographic appraisal of an automatic approach for detecting new word senses», Proceedings of eLex, Tallinn, Estonie.

GIORA Rachel, 2003, On Our Mind. Salience, Context and Figurative Language, Oxford, Oxford University Press.

Grutschus Anke, Kern Beate, Tutin Agnès, 2013, "La polarité du lexique de l'affect. Perspective combinatoire et contrastive», Cartographie des émotions. Propositions linguistiques et sociolinguistiques, Paris, Presses Sorbonne nouvelle, p. 85-96.

HoEY Michael, 2003, «Lexical priming and the properties of text», http://www.monabaker.com/tsresources/LexicalPrimingandthePropertiesofText.htm (consulté le 10 mars 2015).

LE BART Christian, 2009, "Les présidentiables de 2007 entre proximité et surplomb. Nicolas Sarkozy et Ségolène Royal vus par Libération », Mots. Les langages du politique, no89, 2007. Débats pour l'Élysée, p. 31-45.

MCCALL Leslie, 2005, "The complexity of intersectionality», Signs, n³0 (3), p.17711800.

Moureaud Valérie, 2008, «La labellisation des présidentiables en France. Étude de cas d'une légitimation politico-médiatique », Politique et sociétés, nº 27 (2), p.161-189.

PARTINGTON Alan, 2004, «Utterly content in each other's company. Semantic prosody and semantic preference », International Journal of Corpus Linguistics, n 9, p. 131-156.

RABATEL Alain, 1998, La construction textuelle du point de vue, Lausanne, Delachaux et Niestlé.

RASTIER François, 2011, La mesure et le grain, Paris, Honoré Champion.

Rezsohazy Rudolf, 2006, Sociologie des valeurs, Paris, Armand Colin.

SINCLAIR John, 2004, "The search for units of meaning ", Trust the Text. Language, Corpus and Discourse, Londres - New York, Routledge, p. 24-48. 